\title{
Un héroe polaco en las relaciones de sucesos. Memoria e historia editorial de una liberación de esclavos cristianos en el Mediterráneo (1628) \\ A Polish Hero in the "relaciones de sucesos". Memory and Editorial History of the Liberation of Christian Slaves in the Mediterranean (1628)
}

\section{Manuel Garrobo Peral}

Università degli Studi di Verona

ITALIA

manuel.garroboperal@univr.it

[Hipogrifo, (issn: 2328-1308), 9.1, 2021, pp. 373-393]

Recibido: 28-02-2021 / Aceptado: 14-04-2021

DOI: http://dx.doi.org/10.13035/H.2021.09.01.22

Resumen. El artículo se propone dar cuenta de la hazaña de Marek Jakimowski y de trazar la historia editorial de las relaciones de sucesos que la celebraron en 1628, concretamente en Italia, España, Alemania y la República de las Dos Naciones (Lituania y Polonia). Por medio de diversas fuentes bibliográficas trataremos de acercarnos al acontecimiento histórico intentando reconstruirlo y aportando los suficientes datos como para asentar nuevas bases sobre este hecho con el fin de seguir contribuyendo a su estudio.

Palabras clave. Jakimowski; relaciones de sucesos; historia editorial; liberación de esclavos; cristianismo.

Abstract. The article aims to give an account of the feat of Marek Jakimowski and to trace the editorial history of the documents that celebrated it in 1628, specifically in Italy, Spain, Germany and the Republic of the Two Nations (Lithuania 
and Poland). Through various bibliographic sources we will try to get closer to the historical event, trying to reconstruct it and providing enough data to establish new bases to its study.

Keywords. Jakimowski; Event relationships; Editorial history; Slave liberation; Christianity.

\section{INTRODUCCIÓN Y MÉTODO}

El nombre de Marek Jakimowski aún resuena en algunas fuentes dedicadas a la historia sobre el cautiverio cristiano en el Mediterráneo durante los siglos XVI-XVII, especialmente en las polacas e italianas ${ }^{7}$. No es extraño, pues al consultar las relaciones de sucesos podemos observar que la publicación sobre esta temática aumentó considerablemente a finales del siglo XVI y principios del XVII . Marek Jakimowski es el protagonista en una relación romana de 1628 que será objeto de varias ediciones y traducciones. En el texto aparece retratado como un verdadero héroe cristiano que se encarga de liberar a más de doscientos esclavos correligionarios de la tiranía y esclavitud de tres galeras otomanas lideradas por Kassimbech, el por entonces gobernador de Damieta y Rossetto en Egipto. Como es natural, el evento despertó gran interés en los centros editoriales cristianos del momento y la noticia se difundió por la Europa católica durante el mismo año con el claro objetivo de alimentar la propaganda en contra de cualquier pretensión de la Sublime Puerta sobre aguas católicas.

En este trabajo me encargaré de esclarecer dicho evento desde un punto de vista histórico y editorial que permitirá, por lo menos, establecer un panorama general y algunas hipótesis y posibilidades para la reconstrucción de los hechos a través de los relatos que encontremos. Tratar con relaciones de sucesos o con cualquier documento histórico no implica considerar su naturaleza como un fósil que conviene rescatar y cuidar en nuestro «regreso intermitente» al pasado, sino que también resulta necesario avanzar hacia nuestro presente, una vez alcanzada la esencia del fenómeno histórico, es decir, su causalidad y su contigüidad con otros testimonios menos, igual o más relevantes, puesto que ningún hecho se da con iniciativa propia. De este modo, el método que deseamos aplicar aquí es claramente presentista, ya que trataremos, siempre que sea posible, de aproximar la memoria y fenomenología del evento al lector y/o investigador actual.

1. Tanto en el ámbito italiano, como en el español varios autores tratan el suceso: ver Bilinski (1980), Ryba (2014), Polczynski (2014), Ciappelli (2017) o Varriale (2020), que usaremos para nuestro estudio. Además, la SIERS (Sociedad Internacional para Estudio de Relaciones de Sucesos) ha dedicado dos sesiones de sus encuentros trienales a relaciones de sucesos sobre cautiverio y las escaramuzas en el Mediterráneo (ver las actas de Cagliari en 1998 y Trento en 2016). Ver también Chaulet, 2017.

2. Basta consultar CBDRS (Catálogo y Biblioteca Digital de Relaciones de Sucesos) de la Universidade da Coruña y en su búsqueda avanzada marcar como periodo 1580 a 1630 para encontrar unas 538 relaciones sobre acontecimientos militares, políticos y religiosos. 
Para facilitar el adecuado seguimiento de este trabajo, hemos estructurado el discurso en cuatro partes. En primer lugar, ofrecemos una descripción y contexto precisos del evento narrado en las relaciones que hemos encontrado disponibles. En segundo lugar, aportamos los datos esenciales de cada testimonio editorial. Nos centramos en cada uno de ellos, especialmente en los italianos y el castellano (debido a que hemos podido encontrar más información bibliográfica sobre ellos), así como los agentes implicados y la red de relaciones editoriales y comerciales entre España e Italia. De esta forma procuraremos conocer mejor cómo la cultura aurisecular, en especial la editorial, funcionaba en lo que respecta a flujos comerciales y económicos. En tercer lugar, dedicaremos un apartado a otras posibles versiones editoriales del fenómeno y a la problemática cronológica que suponen. Por último, ofrecemos unas conclusiones y un listado de referencias bibliográficas.

\section{DESCRIPCIÓN Y CONTEXTO DEL EVENTO}

Para establecer los datos históricos esenciales del acontecimiento hemos decidido responder al método clásico de las 7W. En este sentido, intentaremos cubrir todos los espacios que giran en torno al suceso sin dejar nada atrás. Pondremos la atención exclusivamente en la relación que narra por primera vez (o al menos, es el documento pionero con el que contamos) el suceso y luego será el turno, en el siguiente apartado, de sus variantes traductológicas.

El título indica que el evento trata «della conquista della galera capitana d'Alessandria nel porto di Metellino per opera del capitano Marco Jakimowski». Efectivamente, así es. Todas las relaciones confluyen en un mismo punto y es que, al detenerse la Galera Capitana en Mitilene (Lesbos) para el descanso de Mehmet y Kassimbech, Jakimowski aprovecha la ocasión para preparar un motín por sí mismo y luego junto a sus compañeros cuando se dan cuenta de que es una buena idea, ya que antes habían intentado disuadirle de sus planes (Stefano Satanousqui y Giovanni Stolcina, concretamente). Tras matar a algunos enemigos, Marek toma el control del barco y todos huyen con la galera hacia la Península Itálica. Las galeras turcas de conserva persiguen a los sublevados durante un tiempo, pero finalmente abandonan su empresa. Marek y los cristianos liberados pasan por diversos lugares hasta llegar a Roma, donde son recibidos por el papa Urbano VIII y otras autoridades. Marco es nombrado caballero del «Speron d'Oro» (Espuela de Oro) y se casa con una de las cautivas llamada Caterina ${ }^{3}$. Estos son, en resumen, los hechos que quedan fijos en cada una de las ediciones que hemos encontrado. En el apartado siguiente profundizaremos en ellos para revelar diferencias, semejanzas y contradicciones.

3. L'Ordine della Milizia Aurata o dello Speron d'Oro (Espuela de Oro) es una orden caballeresca pontificia cuya condecoración se gana a través de grandes hazañas en defensa o en el seno de la Iglesia Católica. Su origen es anterior al siglo XVI. Algunos miembros destacados fueron Rafael Sanzio (1483-1520) o Tiziano (1488/1490-1576). 
Jakimowski es, sin duda, el foco central de la relación. Marco fue un noble del reino polaco-lituano que participó en la batalla de Cécora $(1620)^{4}$, contra los turcos, fue hecho prisionero y comprado por Kassembeg (o Kassimbech), gobernador de Damieta y Rosetta en Egipto, y se le asignó su galera siendo uno de los esclavos con mayor libertad de movimiento dentro de ella ${ }^{5}$. Tras liberarse, se casaría y volvería a Cracovia con su nueva mujer Katarzyna. Su heroicidad resonó en toda Europa gracias la publicación de varias ediciones, como acabamos de comentar. De hecho,

The event was commemorated by the Venetian painter Tommaso Dolabella, active in the court of King Sigismund III Vasa (1566-1632) and his son, King Wladyslaw IV Vasa (1595-1648), whose work depicted the marriage of Marek and Katarzyna. During the 19th century, following the partitioning of Poland-Lithuania by its neighbors, Russia, Prussia, and Austria, Jakimowski appeared in nationalist literature, though, in a potent reminder of the malleability of history in support of nationalist projects, Jakimowski's struggle against his Ottoman captors was employed not to symbolize the struggles of Poles against the Sultan, but rather their struggles against the Sultan's mortal enemy: the Tzar of Russia'.

El apodo de «Padoliese russiano», tal y como lo describe Marnavitio en la relación romana de 1628, nos remite directamente a dos territorios que apuntarían a su origen. El primer gentilicio se refiere a la región de Podolia (Polonia). Respecto al segundo, a primera vista podríamos pensar que estuviera totalmente emparentado con las personas provenientes de Rusia al contar con una clara aproximación léxica con «russo» y sin descartar que pueda tratarse de una variante gentilicia de esta. Sin embargo, podríamos apuntar, como hipótesis etimológica, que su conexión tiene que ver más con la etnia rutena, ya que Marco provenía de una tierra de frontera que, en su momento, pertenecía al reino de Polonia y ahora es parte de Ucrania. Esta región, denominada Rutenia, y coincidente con la región de Podolia por entonces, adopta varios gentilicios como rutenos, rusinos, cárpato-rusos o rusniacos, por lo que Marnavitio estaría siendo preciso al indicar el origen de nuestro héroe?.

Jakimowski se convirtió posteriormente en el combustible perfecto para contextos emergentes del nacionalismo polaco en el siglo XIX. Varios historiadores polacos se basaron en su hazaña como Grabowski en 1840 o Milewski en 18488. La construcción y difusión del relato, en este sentido, trascendió a los nacionalismos decimonónicos.

5. Marnavitio indica que él y sus dos compañeros «camminavano liberi per il vascello a fine di servizi pubblici di quello» (Relazione della conquista della galera capitana d'Alessandria nel porto di Metellino per opera del capitano Marco Jakimowski..., p. 4.

6. Polczynski, 2014.

7. La región histórica de Rutenia pertenece en la actualidad a Ucrania, pero en el siglo XVII era un territorio que contaba también con regiones de Eslovaquia y Polonia. Robert Magosci (1995) dedica un artículo a la cuestión rusiana y corrobora estos problemas de nomenclatura en la denominación poblacional, además de los de identidad cultural.

8. Ryba, 2014. 
Además, conviene indicar otras personalidades que se indican en la relación romana. En primer lugar, el Cardenal Giovanni Doria (1573-1642), arzobispo de Palermo y Tesalónica al servicio del imperio español, que es mencionado en la dedicatoria inicial. En segundo lugar, Mehmet, capitán de las cuatro galeras. Sabemos además que lo fue durante cuatro años, que reprimió previamente una incursión de cosacos y que se retiró aquel invierno «alla cura della sua carica» para luego embarcar al juez de Alejandría y a su mujer y dirigirse a Mitilene. En tercer lugar, el cardenal Cosimo de Torres (1584-1642), protector de los polacos católicos en Roma que, según Marnavitio, recibe la donación de las banderas de la Galera Capitana como muestra de afecto y respeto. En cuarto y último lugar, se mencionan los nombres de Carlo (1562-1630) y Taddeo Barberini (1603-1647), duque de Monterotondo y príncipe de Palestrina (Lazio) respectivamente, y miembros de la familia más poderosa durante la primera mitad del siglo XVII en los Estados Pontificios. Marnavitio indica que también reciben banderas como donación.

En 1628 se publican por lo menos las cinco relaciones que hemos recuperado para este estudio. Este es un año más de contención del Imperio Otomano en Europa, a pesar de su reconocida debilidad tras la inesperada derrota en Lepanto (1571). Sus incursiones, especialmente marítimas, continuaban siendo un fenómeno totalmente común y por esta razón las relaciones de sucesos sobre lo que sucedía en los mares eran bastante recurrentes. Siendo Viena baluarte central y foco difusor de noticias sobre el belicismo turco desde finales del XVI y a lo largo de todo el XVII, los flujos comerciales de relaciones crecieron en ciudades como Barcelona, Milán, Génova, Lyon, Roma o Florencia9 ${ }^{9}$ donde florecieron centros editoriales de propaganda antiturca ${ }^{10}$. En este sentido, en palabras de Redondo,

[Estas relaciones difundieron] la imagen de una alteridad musulmana rechazada, arrogante, cruel, viciosa y a veces ridícula, contra la cual había que luchar y que era necesario vencer para conseguir el triunfo de la religión católica. Han contribuido, de tal modo, a mantener el espíritu de cruzada y el sueño mesiánico del desplome del imperio del Sultán de Constantinopla y de la próxima llegada del reino de Cristo sobre la tierra ${ }^{11}$.

La narración detalla los sucesos que tienen lugar en el viaje desde Mitilene (Lesbos) a Cracovia (República de las Dos Naciones). En el siguiente mapa resumimos la supuesta trayectoria de Kassimbech (con una línea negra) y luego la de Jakimowski tras su liberación (con una línea roja) y su regreso a Cracovia (con una línea azul), siempre considerando el testimonio romano. También hemos añadido tres localizaciones que nos parecen esenciales: Baro (lugar de nacimiento de Jakimowski), Cécora (territorio de frontera donde se produjo en 1620 la derrota polaca 
frente a la incursión de los crimeanos) y el propio estuario del Dniéper, donde suponemos, o al menos cerca de este, que se pudo producir la incursión de los cosacos reprimida por Mehmet antes de dirigirse a Constantinopla12:

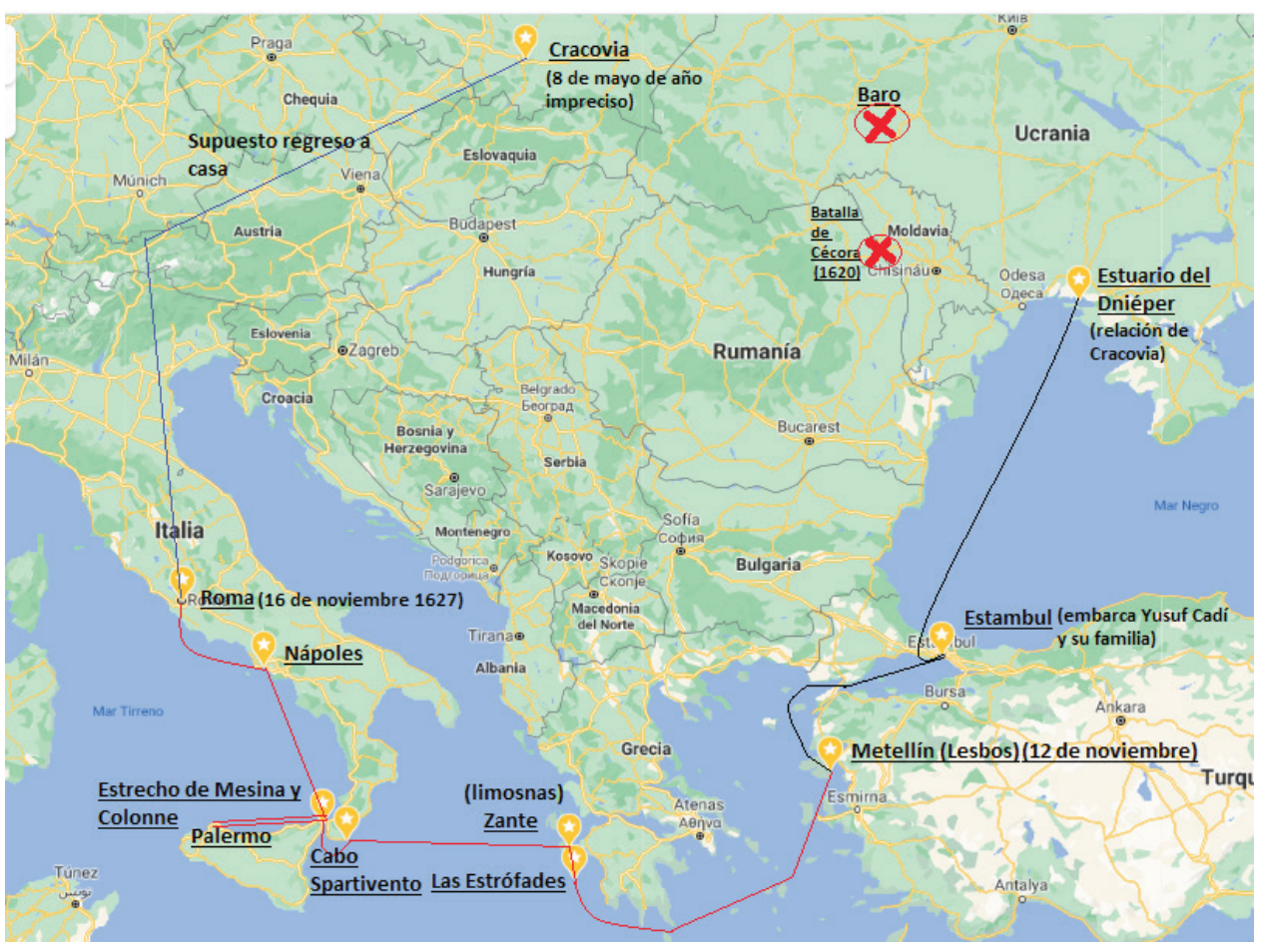

El porqué de la extraordinaria difusión de esta historia es el núcleo de nuestro trabajo y trataremos de ofrecer respuestas con la comparación entre los testimonios obtenidos. Lo que nos queda claro, de todos modos, es la intención propagandística e ideológica a favor de la Cristiandad frente a su enemigo principal, que subyace en cada uno de los escritos ${ }^{13}$. En cuanto a un porqué interno a la relación, es también evidente que la ocasión que se les brindó a Jakimowski y a sus compañeros no podía ignorarse, al parecer, pues nada iba ni fue en su contra. Esto también nos hace sospechar un poco de la verdad del motín. Dirigirse a aguas italianas y españolas era lo más lógico para encontrar nuevas alternativas a la escla-

12. Santa Cruz, en la traducción castellana, omite gran parte de esta geografía tan esencial para trazar la posible ruta del polaco, pero también la de Kassimbech con el contexto introductorio anterior (que tampoco figura en la relación barcelonesa) y la referencia a la vuelta de Marco el 8 de mayo gracias a la indicación en la edición cracoviana.

13. Según Ciappelli (2017, p. 137), «lo scopo insieme edificante e propagandistico dal punto di vista politico era evidente, sia nel linguaggio, sia nelle sottolineature». [...] «Le rimanenti numerosissime versioni castigliane di imprese di mare toscane stampate entro il 1639 si giustificano o perché le azioni toscane sono state condotte insieme a navi spagnole (galere di Napoli o Sicilia) [...] o perché le galere toscane stavano navigando in acque spagnole» (2017, p. 148). Efectivamente, la narración cuenta el bizantino recorrido desde Lesbos a Roma pasando por Sicilia, Nápoles y otras pequeñas localidades. 
vitud $^{14}$. La edulcoración del relato convierte la causalidad de los esclavos en pura casualidad, esto es, coincidencia encadenada de acontecimientos «otorgados» por la providencia divina hacia la obtención de la ansiada y merecida libertad. Debemos sospechar, sin embargo, de la falta total de logística y prevención otomana respecto a posibles motines, tal y como se deja claro en la relación romana.

En cuanto al cómo, podríamos resumirlo con la astucia de Jakimowski, pero no bastaría para aproximarnos de forma más verosímil al hecho histórico. Son también los compañeros, por supuesto, los que hacen posible que el suceso tenga éxito. Presumimos, por tanto, que hubo una coordinación entre los esclavos cuyo liderazgo recaería en Jakimowski, pero, a no ser por cuestiones fortuitas, rechazamos la idea de que haya sido solo él quien haya sido capaz de realizar dicha empresa. Los medios principales para la liberación son los siguientes:

A) Un leño que Jakimowski roba para matar al cocinero de la Galera Capitana ${ }^{15}$.

B) Las armas de la popa que Jakimoswki reparte con todos los esclavos tras derrotar en combate a un soldado griego renegado.

C) Los remos para hacer zarpar el barco del puerto de Mitilene.

D) Clima favorable (gracias a la providencia, según Marnavitio y el resto de auto-

res) a la Galera Capitana que detiene la persecución de las otras galeras.

\section{TESTIMONIOS Y DIFUSIÓN}

Ahora nos encargaremos de aportar los datos fundamentales (título, autor, lugar de publicación, impresor, mecenas, tamaño, número de páginas, letra y localización) de cada uno de los testimonios encontrados y estableceremos una comparación estructural (formal y de contenido) y contextual que nos ofrece cada uno de ellos.

\subsection{Relación romana de 1628}

Título: Relazione della conquista della galera capitana d'Alessandria nel porto di Metellino per opera del capitano Marco Jakimowski, schiavo in detta Gallera. Con liberatione di 220 schiavi cristiani.

Autor: Marco Tomeo Marnavitio.

Lugar de edición: Roma.

Impresor: Ludovico Grignani.

Dedicatario: Conde Escipión d'Acquaviva y Aragón.

14. Como nos señala Varriale (2020, p. 84), liberar cautivos «se convirtió en una de las obras más preciadas a los ojos de Dios», por lo que «surgieron decenas de cofradías y congregaciones, tanto religiosas como laicas, que se ocupaban de liberar a los correligionarios en manos de musulmanes»; de ahí también que liberarse sin rescate o sin negociación entre las partes implicadas supusiese todo un reto y una gran heroicidad. Podrían, en efecto, haber recurrido a un rescate, pero optaron por una vía más arriesgada.

15. Curiosamente solo en la relación polaca Marco coge tres mazas en lugar de un leño para defenderse del cocinero. 
Formato: $8{ }^{\circ}$.

Páginas: 7 .

Letra: redonda y cursiva

Signatura: b. sygn.

Localización: Digital Library of Polish and Poland-Related News Pamphlets from the 16th to the 18th Century cuyo identificador es el núm. 381.

El proceso editorial arranca con el acuerdo entre un impresor y un obispo: Ludovico Grignani (1586-1651) y Marco Tomeo Marnavitio (¿?- ¿?). Grignani fue impresor italiano muy conocido tal y como lo declara la enciclopedia Treccani. Sabemos que en el año 1628 «si trasferì nuovamente e impiantò la sua tipografia in una casa di proprietà del Collegio degli orfani a piazza di Pietra» y que la década de los 20 «divenne subito famoso per la stampa di avvisi italiani», de ahí que sea responsable de la publicación de este caso tan sonado en Europa. También era muy conocido por ser impresor de importantes textos musicales y más tarde de opúsculos populares, obras de autores locales, textos teatrales y antologías poéticas. Parece, por tanto, que resultó ser un hombre muy demandado en la imprenta romana de su tiempo. Incluso el propio papa Urbano VIII le encargó una nueva edición del Breviario romano ${ }^{16}$. Por lo que respecta a Marnavitio (su nombre está italianizado y el verdadero es Marco Tomko Marnavič) fue «nipote di Ivano Tomko Marnavič, vescovo di Bosnia (1579-1637)» ${ }^{17}$. La información bibliográfica que encontramos se centra más en el segundo, pero al mismo tiempo nos revela el grado de importancia social con el que contaba esta familia en el momento en que el sobrino publicó la relación de sucesos en Roma. De hecho, al obispo se le considera, en anotaciones biográficas como la que presenta Treccani, un historiador falsario, puesto que publicó, alrededor de 1619, la Vita lustiniani, donde defendió la tesis de que el famoso emperador tenía orígenes eslavos. Ante esta vena nacionalista, no nos extraña que acogiese tan bien la noticia sobre un héroe polaco que, gracias a su astucia e ingenio, consiguió salvar a más de doscientos esclavos cristianos. Por lo que respecta a Marnavitio sobrino, no contamos con información precisa sobre su vida, salvo que se encargó de escribir la hazaña por sugerencia de su tío y que «doveva a quell'epoca trovarsi a Roma, all'Ospizio di S. Gerolamo degli Schiavoni, in cui furono ospitati i prigionieri liberati dalla Galera» ${ }^{18}$.

16. Sabemos que su producción bibliográfica fue inmensa y sería muy interesante poder localizar, si lo hubo, un catálogo de todo lo que produjo en vida. No obstante, existen algunas bases de datos donde podemos encontrar reunidas algunas de sus obras, como es el caso de la Biblioteca Nacional de Francia: aquí se conserva una miscelánea de relaciones, cartas y tratados de diverso tipo publicada entre 1625 y 1647. Se agrupan en obras textuales, mixtas y musicales.

17. Bilinski, 1980, p. 77.

18. Bilinski, 1980, p. 80 


\subsection{Relación florentina de 1628}

Título: Relazione della conquista della galera capitana d'Alessandria nel porto di Metellino per opera del capitano Marco Jakimowski, schiavo in detta galera. Con liberazione di dugento schiavi christiani...

Autor: Marco Tomeo Marnavitio.

Lugar de edición: Roma y Florencia.

Impresor: Francesco Onofri.

Dedicatario: Quizás el mismo conde Escipión al ser Onofri uno de sus impresores personales.

Formato: $4 .{ }^{019}$.

Páginas: 8.

Letra redonda y cursiva.

Signatura: Sygn. $A^{\wedge} 4^{\wedge}$.

Localización: Digital Library of Polish and Poland-Related News Pamphlets from the 16th to the 18th Century cuyo identificador es el núm. 382.

En cuanto a Francesco Onofri, podemos ofrecer alguna información de relevancia. En las fuentes que hemos encontrado constatamos que se trató de un impresor florentino muy conocido durante el siglo XVII, pues se le denomina «stampatore granducale» $\mathrm{y}$ «di tutti i magistrati», por lo que queda clara una relación sólida con la alta nobleza de Toscana y el gran ducado (1569-1859) y nos hace suponer que sirvió a Cosimo II (activo entre 1590-1621) y Ferdinando II (activo entre 1610-1670) de Médici20.

Además, podemos trazar un largo periodo de actividad a través de las fuentes primarias que tenemos: de 1628 con la relación florentina hasta 1681 con el extracto medicinal21. También la Biblioteca Statale Isontina di Gorizia viene publicada una lista de tipógrafos en siglo XVII y se indica que su actividad empieza en 1631 y termina en 1680. La fuente principal es la British Library. Esto crea, no obstante, una contradicción en la información que aparece en las obras mencionadas y aquella perteneciente a las fuentes secundarias.

19. Nos extraña la elección de este formato, al no coincidir en número con la foliación. Probablemente sea un formato en octavo.

20. Su producción bibliográfica es extensa. En los siguientes enlaces podemos encontrar algunas obras impresas suyas como en Treccani, Internet Culturale. También se menciona su actividad editorial en los Annali della tipografia fiorentina de Lorenzo Torrentino (1819) (p. 265), en Della storia e della ragione d'ogni poesia. Volume quarto de Francesco Saverio Quadrio (1749) o en Gli scrittori d'Italia, cioè notizie storiche e critiche... de Mazzuchelli (1758).

21. Existe un estudio histórico reciente sobre la tipografía de Onofri en colaboración con Angelo Raimondi. Se ha publicado en 2006 en los Annali Aretini por Elisa Boffa. 


\subsection{Relación-traducción alemana (1628)}

Título: Verwunderlicher Seehafenkrieg, das ist wahhafte Neue Zeitung wasmassen ein geborener Pole, aber gefangener Sklave, eine türkische Hauptgaleere durch seine Tapferkeit bewältigt und mit grossem Reichtum in die Christenheit gebracht hat.

Lugar de edición: Alemania. Asumimos que haya salido de algún lugar de impresión en terreno católico (Augsburgo, Núremberg, Múnich...), es decir, en la región bávara; o incluso de Viena, ciudad, como hemos dicho, de contención turca en el siglo anterior y activa en relaciones de sucesos propagandísticas.

Formato: $4 .{ }^{022}$.

Páginas: 9.

Letra: gótica.

Signatura: Sygn. $A^{\wedge} 4^{\wedge}$.

Localización: Digital Library of Polish and Poland-Related News Pamphlets from the 16th to the 18th Century cuyo identificador es el núm. 1804.

No hemos encontrado ninguna información más respecto a esta relación. Estamos abiertos a nuevas sugerencias o aclaraciones.

\subsection{Relación-traducción polaca (1628)}

Título: Opisanie krótkie zdobycia galery aleksandryjskiej w porcie Metellino za sprawą kapitana Marka Jakimowskiego.

Lugar de edición: Cracovia.

Formato: $4{ }^{\circ}$.

Páginas: 6.

Letra: redonda, cursiva y gótica.

Signatura: Sygn. $A^{\wedge} 4^{\wedge}$

Localización: Digital Library of Polish and Poland-Related News Pamphlets from the 16th to the 18th Century cuyo identificador es el núm. 380.

Desconocemos más datos de los que nos proporciona la Digital Library y por lo que hemos leído, existen algunos autores polacos que han rescatado la historia en ediciones modernas como la de Boleslaw Śląski de 1927 en conmemoración del evento que, supone, ocurrió en 1627. Este autor deja claro en su prólogo al lector que «Polska tedy opowieść o Jakimowskim jest tylko przekładem»²3 ('la historia polaca sobre Jakimowski es solo una traducción'), una traducción del italiano al polaco con algunas variaciones textuales. Sabemos también, gracias a este autor,

22. De nuevo, la misma falta de lógica entre páginas y formato.

23. Śląski, 1927, p. 5. 
que el acontecimiento también lo consideró Ambroże Grabowski en 1840 en su Staroż Hist Polsk (concretamente en el volumen 1) y también tratado en poesía por Aleksander Groza ${ }^{24}$. Por otra parte, Szymon Starowolski en sus Principios del acuerdo militar, escrito en latín en 1639, menciona que Jakimowski no perseguía ningún objetivo ulterior a su liberación, solo llegar a tierras cristianas y vivir el resto de su vida tranquilo con Katarzyna ${ }^{25}$. Además, Śląski nos indica que «es un misterio dónde este noble de Podolia ganó tanta experiencia en la navegación para poder conducir un barco por el mar y manejarlo sin dificultad durante una tormenta» ${ }^{26}$. Por tanto, aunque contemos con fuentes y con estudiosos sobre el asunto, existen todavía lagunas editoriales, comerciales y biográficas (del héroe) que convendría aclarar.

\subsection{Relación-traducción barcelonesa (18 de julio de 1628). La noticia en España y relaciones editoriales.}

Título: Relacion verdadera del levantamiento, y presa de la Galera Capitana de Alexandria, en el Puerto de Metellin, por industria del Capitan Marco Lachimosch Esclauo de dicha Galera, con libertad de 220. Christianos Esclauos, à 18. de Iulio 1628. Impressa ya en Roma. Traduzida de lengua Italiana en Castellano, por Miguel de Santa Cruz.

Autor: Miguel de Santa Cruz

Lugar de edición: Barcelona

Impresor: Esteban Liberòs

Formato: $4 .^{\circ}$.

Páginas: 2 folios.

Letra: redonda y cursiva.

Localización: Catálogo y Biblioteca Digital de Relaciones de Sucesos (CBDRS) cuyo código es CBDRS 0004447 . Se conserva en la Biblioteca General de la Universidad de Barcelona (B-59/342-77 y B-44/3/5-8) y el otro en la Biblioteca de Cataluña (Fondo Bonsoms 10956).

Ahora es el turno del impresor barcelonés Esteban Liberòs y el traductor Miguel de Santa Cruz. En primer lugar, podemos indicar que se trató de un impresor muy solicitado e importante del momento. Según Delgado Casado en su Diccionario de impresores españoles (s. XV-XVIII), la fecha de actividad de este impresor parte aproximadamente de 1613 a 1633 en Barcelona y luego en 1645 en Murcia27. Con los datos del corpus de relaciones de sucesos traducidas al español de López Poza nos damos cuenta también de la importancia que tuvo, pues «de su imprenta sa-

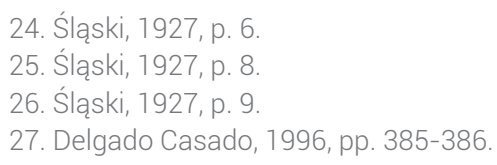


lieron al menos 148 relaciones impresas entre 1615 y 1628»28. Además, sabemos también que imprimió una relación en italiano «para ser exportada en un buque de mercancías al mercado italiano», por lo que se podría confirmar su sólida relación con Italia y su probable interés por las posibilidades económicas de las traducciones de relaciones ${ }^{29}$. Por otra parte, este impresor también es bien conocido por su trabajo editorial en obras de gran importancia como los Sueños... de Quevedo en 1627. En segundo lugar, en relación con Santa Cruz, no hemos encontrado ninguna fuente bibliográfica que lo mencione siquiera en manuales sobre historia de la traducción ${ }^{30}$ y, por supuesto, agradeceríamos cualquier referencia al respecto. De todos modos, no nos puede extrañar que a veces nos topemos con terrenos baldíos en la investigación de traductores auriseculares o simplemente en referencia a la autoría de relaciones de sucesos. En este sentido,

Indicar el nombre del traductor parece excepcional, y por lo común parecen personas que realizan este trabajo ocasionalmente, no de manera profesional. Con cierta frecuencia son personas vinculadas a órdenes religiosas ${ }^{31}$.

Por otra parte, podemos también indicar, a partir de los datos el CBDRS, que la temática sobre contiendas en el mar fue muy común a partir del primer decenio del siglo XVII (1616-1628) y especialmente explotada en la imprenta de Liberòs. Contamos con 8 relaciones de sucesos salidas de esta imprenta que se enmarcan en la tipología de acontecimientos políticos y religiosos. Podemos dividirlas en dos subtipos temáticos:

-Victoria y/o presa de galeras turcas por católicos ${ }^{32}$.

- Hechos de cautivos ${ }^{33}$

El primer grupo de relaciones nos revela de nuevo la sólida relación comercial que había entre la imprenta de Liberòs y los reinos italianos, puesto que tres de ellas (0005036, 0005087 y 0002611) hacen referencia a hechos realizados por el Gran Ducado de Toscana y el Virreinato de Sicilia. El primero, como sabemos, tiene una imprenta a su servicio, que es la de Onofri (Florencia), por lo que podríamos establecer como hipótesis un contacto claro entre Barcelona e Italia (Gran Ducado de Toscana y Roma).

32. Añadimos las signaturas correspondientes con sus enlaces: $\underline{0000337}, \underline{0002611}, \underline{0005324}, \underline{0005035}$, $\underline{0005499}, 0005036$.

33. Encontramos una relación sobre una cristiana cautiva en Argel y la conversión de tres musulmanas: https://www.bidiso.es/CBDRS/ediciones/BDRS0000509/153/ejemplar/5003. 


\subsection{Estudio comparativo de las propiedades textuales de cada relación}

Vamos a adoptar en este apartado una serie de factores de comparación que nos harán el camino más fácil para apreciar las discordancias entre las relacionestraducciones con respecto a la original. De esta manera, para hallar las diferencias y las semejanzas, debemos tener en cuenta las recurrencias temáticas y formales principales de cada escrito. Las líneas que nos sirven como base para nuestra comparación son las siguientes:

a) Dedicatorias.

b) Estilo general.

c) Descripción del héroe.

d) Cuestiones geopolíticas.

e) Marco temporal del viaje y editorial.

f) Números.

g) Geografía del viaje.

\begin{tabular}{|c|c|c|c|c|}
\hline \multicolumn{5}{|c|}{ DEDICATORIAS } \\
\hline Roma & Florencia & Cracovia & $\begin{array}{l}\text { Relación en } \\
\text { alemán }\end{array}$ & Barcelona \\
\hline $\begin{array}{l}\text { Dedicatoria extensa } \\
\text { a Escipión Diacceto } \\
\text { d'Acquaviva } \\
\text { Aragona. }\end{array}$ & $\begin{array}{l}\text { Copia exacta de la } \\
\text { dedicatoria, al ser } \\
\text { el conde dueño y } \\
\text { señor de Toscana } \\
\text { y, por tanto, de } \\
\text { Florencia. }\end{array}$ & $\begin{array}{l}\text { Elisión completa. } \\
\text { Quizás por ser } \\
\text { ajena a territorios } \\
\text { polaco-lituanos. }\end{array}$ & $\begin{array}{l}\text { Elisión completa. } \\
\text { Quizás por ser } \\
\text { ajena a territorios } \\
\text { alemanes. }\end{array}$ & $\begin{array}{l}\text { Elisión completa. } \\
\text { Quizás por ser } \\
\text { ajena a territorios } \\
\text { hispánicos. }\end{array}$ \\
\hline
\end{tabular}

\begin{tabular}{|l|c|c|c|c|}
\hline \multicolumn{7}{|c|}{ ESTILO GENERAL } \\
\hline Roma & Florencia & Cracovia & $\begin{array}{c}\text { Relación en } \\
\text { alemán }\end{array}$ & Barcelona \\
\hline
\end{tabular}

Propagandístico e ideológico a favor de la Iglesia Católica. En primer lugar, las dedicatorias de la relación romana y florentina revelan el carácter obsecuente de los humanistas italianos del momento ante sus mecenas. En segundo lugar, la narración en todas las ediciones contiene tintes épicos y caballerescos. La que más destaca respecto a esto último es la barcelonesa, al situar la acción de forma atemporal (sin explicación contextual) y retratando a Marco como el verdadero héroe cristiano que satisface deseos mesiánicos frente al Imperio Otomano. 


\begin{tabular}{|c|c|c|c|}
\hline \multicolumn{4}{|c|}{ DESCRIPCIÓN DEL HÉROE } \\
\hline Florencia & Cracovia & $\begin{array}{l}\text { Relación en } \\
\text { alemán }\end{array}$ & Barcelona \\
\hline $\begin{array}{l}\text { Descripción más o menos realista del } \\
\text { héroe. } \\
\text { Recibe heridas en la cabeza y el hombro y } \\
\text { sangra. } \\
\text { Personalidad determinada y con } \\
\text { liderazgo. }\end{array}$ & $\begin{array}{l}\text { Descripción más o } \\
\text { menos realista del } \\
\text { héroe. } \\
\text { Recibe heridas } \\
\text { leves durante el } \\
\text { motín. } \\
\text { Personalidad } \\
\text { determinada y con } \\
\text { liderazgo. }\end{array}$ & $\begin{array}{l}\text { Misma que en la } \\
\text { relación original. }\end{array}$ & 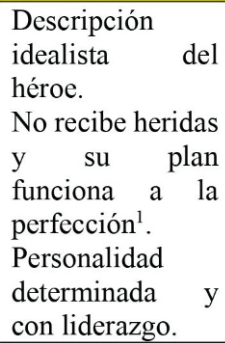 \\
\hline
\end{tabular}

\section{CUESTIONES GEOPOLÍTICAS}

\begin{tabular}{|c|c|c|c|c|}
\hline Roma & Florencia & Cracovia & $\begin{array}{l}\text { Relación en } \\
\text { alemán }\end{array}$ & Barcelona \\
\hline \multicolumn{4}{|c|}{$\begin{array}{l}\text { Contextualización precisa de la situación del Imperio Otomano tras el Concilio de } \\
\text { Trento (1545-1563) y tras Lepanto (1571): escaramuzas marítimas, negocio de } \\
\text { esclavos en el Mediterráneo. Se añade un dato interesante: el cambio en los } \\
\text { prisioneros del Imperio Otomano. Ahora provienen solo de territorios de Europa del } \\
\text { Este porque Europa Occidental se ha reforzado. De ahí que contemos con solo } 3 \\
\text { griegos, } 2 \text { ingleses, } 1 \text { italiano y el resto de Rusia. }\end{array}$} & $\begin{array}{l}\text { Omisión total de } \\
\text { la situación } \\
\text { geopolítica en } \\
\text { 1628. Santa Cruz } \\
\text { pasa } \\
\text { directamente al } \\
\text { evento en sí. }\end{array}$ \\
\hline
\end{tabular}

\begin{tabular}{|c|c|c|c|c|}
\hline \multicolumn{5}{|c|}{ MARCO TEMPORAL DEL VIAJE Y EDITORIAL } \\
\hline Roma & Florencia & Cracovia & $\begin{array}{l}\text { Relación en } \\
\text { alemán }\end{array}$ & Barcelona \\
\hline $\begin{array}{l}\text { Mehmet } \\
\text { desembarca en } \\
\text { Metellín el } 12 \text { de } \\
\text { noviembre de } \\
1627 \text {. } \\
\text { Llegan a Roma el } \\
16 \text { de febrero de } \\
1628 \text {. } \\
\text { Se publica la } \\
\text { relación el } 24 \text { de } \\
\text { febrero de } 1628 \text {. }\end{array}$ & $\begin{array}{l}\text { Sigue el mismo } \\
\text { marco temporal } \\
\text { que la relación } \\
\text { romana. } \\
\text { Misma fecha de } \\
\text { publicación que la } \\
\text { relación romana. }\end{array}$ & $\begin{array}{l}\text { Sigue el mismo } \\
\text { marco temporal } \\
\text { que la relación } \\
\text { romana. Se añade } \\
\text { que Jakimowski } \\
\text { regressa a } \\
\text { Cracovia el } 8 \text { de } \\
\text { mayo de } 1628 . \\
\text { Esta relación se } \\
\text { publica en } 1628 \text {. }\end{array}$ & $\begin{array}{l}\text { Sigue el mismo } \\
\text { marco temporal } \\
\text { que la relación } \\
\text { romana. } \\
\text { Esta relación se } \\
\text { publica en } 1628 .\end{array}$ & $\begin{array}{l}\text { Mehmet } \\
\text { desembarca el } 18 \\
\text { de julio de un año } \\
\text { impreciso. } \\
\text { Llegan a Roma el } \\
25 \text { de agosto de } \\
\text { un año impreciso. } \\
\text { Se publica esta } \\
\text { relación el } 18 \text { de } \\
\text { julio de } 1628 .\end{array}$ \\
\hline
\end{tabular}

34. Efectivamente, la descripción barcelonesa nada tiene que ver con las anteriores. Marnavitio asegura que Jakimowski ha «ricevuto crudel ferita sopra il capo dalla parte sinistra / un'altra sopra spalla» y luego su estado posterior: «insaguinato come era» (Relazione della conquista della galera capitana d'Alessandria nel porto di Metellino per opera del capitano Marco Jakimowski..., p. 5). El barco tampoco recibe ningún daño en la traducción de Santa Cruz. 


\begin{tabular}{|c|c|c|c|}
\hline \multicolumn{4}{|c|}{ NÚMEROS } \\
\hline Florencia & Cracovia & $\begin{array}{c}\text { Relación en } \\
\text { alemán }\end{array}$ & Barcelona \\
\hline $\begin{array}{l}\mathbf{2 2 0} \text { esclavos cristianos. } \\
\text { El capitán acompañado de } \mathbf{7 0} \text { turcos y } \mathbf{8 0} \\
\text { se quedan en la galera capitana. } \\
\text { Un leño para matar al cocinero. } \\
\mathbf{2 2} \text { turcos condenados al remo y después } \\
\text { liberados. } \\
\mathbf{2 0 0} \text { reales a los monjes de la isla de Zante. } \\
\mathbf{4} \text { mujeres cristianas: Anna, Caterina, } \\
\text { Margarita y una soltera (zitella) que luego } \\
\text { se convierte en cristiana y se hace llamar } \\
\text { Caterina. } \\
\text { Marco llega con } \mathbf{3 5} \text { compañeros a Roma. }\end{array}$ & $\begin{array}{l}\text { Cambios respecto } \\
\text { a la original: } \\
\mathbf{3} \text { mazas o palos } \\
\text { para matar al } \\
\text { cocinero. } \\
\text { Visitan } 9 \text { iglesias } \\
\text { más en Roma. }\end{array}$ & $\begin{array}{l}\text { Cambios: } \\
\text { Algunos palos } \\
\text { (no se determina } \\
\text { el número) para } \\
\text { matar al } \\
\text { cocinero. } \\
\text { No se menciona } \\
\text { el nombre } \\
\text { auténtico de la } \\
\text { última prisionera } \\
\text { y se indica que se } \\
\text { convierte } \\
\text { bautizándose con } \\
\text { el nombre de } \\
\text { Aida }{ }^{2} \text {. } \\
\text { Marco se queda } \\
\text { con } \\
\text { compañeros en } \\
\text { Roma. }\end{array}$ & $\begin{array}{l}\text { Cambios: } \\
\text { 150 pasajeros, } \\
\text { soldados } \\
\text { oficiales en la } \\
\text { galera. No se } \\
\text { especifica el } \\
\text { número de turcos } \\
\text { fuera de la galera } \\
\text { acompañando al } \\
\text { capitán (fol. 2). } \\
\text { Omisión de los } \\
\text { 200 reales. } \\
\mathbf{4} \text { mujeres } \\
\text { cristianas: } \\
\text { Misma } \\
\text { información, } \\
\text { pero se oculta } \\
\text { que Caterina se } \\
\text { llamaba Zitella y } \\
\text { era musulmana. }\end{array}$ \\
\hline
\end{tabular}

35

\begin{tabular}{|c|c|c|c|}
\hline \multicolumn{4}{|c|}{ GEOGRAFÍA DEL VIAJE } \\
\hline Florencia & Cracovia & $\begin{array}{c}\text { Relación en } \\
\text { alemán }\end{array}$ & Barcelona \\
\hline $\begin{array}{l}\text { Río Boristhene, Niéster o Dnper (defensa } \\
\text { de Mehmet ante los cosacos). } \\
\text { Constantinopla (embarca Yusuf Cadí y su } \\
\text { mujer). } \\
\text { Metellín (Lesbos), en el inicio del mar } \\
\text { Egeo. } \\
\text { Puertos de Metellín: Largo, Caramusciali } \\
\text { y Stretto. } \\
\text { Mención a Baro (Podolia) } \\
\text { Las islas Estrófades y la isla Zante. } \\
\text { Colonne } \\
\text { Cabo Spartivento } \\
\text { Costa de Calabria y Reggio. } \\
\text { Palermo } \\
\text { Estrecho de Mesina } \\
\text { Nápoles } \\
\text { Roma } \\
\text { Iglesia de S. Jerónimo de la Ripetta } \\
\text { (Roma). } \\
\text { Iglesia de San Cayo Papa y Mártir } \\
\text { (Roma). }\end{array}$ & $\begin{array}{l}\text { Adiciones: } \\
\text { Cracovia para el } \\
\text { regreso a casa el } 8 \\
\text { de mayo de } 1628 . \\
\text { Omisiones: } \\
\text { Colonne, } \\
\text { Spartivento, costa } \\
\text { de Calabria y } \\
\text { Reggio y Mesina. }\end{array}$ & $\begin{array}{l}\text { Adiciones: } \\
\text { En el tercer folio, } \\
\text { se indica que } \\
\text { Yusuf Cadí se } \\
\text { convierte en juez } \\
\text { de Alejandría, } \\
\text { pero también en } \\
\text { protector de los } \\
\text { territorios entre } \\
\text { el mar Egeo y } \\
\text { Micenas } \\
\text { (Grecia), por lo } \\
\text { que invita a } \\
\text { Kassimbech para } \\
\text { que le traslade a } \\
\text { su nuevo destino. } \\
\text { Omisiones: } \\
\text { Colonne, } \\
\text { Spartivento, } \\
\text { costa de Calabria } \\
\text { y Reggio, } \\
\text { Palermo ýales. } \\
\text { Nápoles }\end{array}$ & $\begin{array}{l}\text { Omisión del río } \\
\text { Niéster, de los } \\
\text { tres puertos de } \\
\text { Metellín, las islas } \\
\text { Estrófades, } \\
\text { Zante, Colonne, } \\
\text { Spartivento y } \\
\text { costa de Calabria } \\
\text { y Reggio. }\end{array}$ \\
\hline
\end{tabular}

35. Esto hace replantearnos una vez más la veracidad de la historia. En el resto de fuentes aparece Caterina, pero nada de Aida. 


\subsection{Posibilidad de otras variantes. Fuentes.}

Hasta ahora hemos presentado las ediciones que hemos podido recuperar en las bibliotecas digitales, pero esto solo es el primer paso hacia una complejidad mayor, pues otras fuentes bibliográficas y documentales apuntan a la existencia de otras relaciones que darían una versión distinta de los hechos:

a) Relato alternativo A. Polczynski en «A Polish Slave's Escape from an Ottoman Galley». Según este historiador, existiría un ejemplar del mismo evento en 1623 titulado La conquista della galera di Alessandria nel porto di Metelline, coll'opera e gran coraggio del Capitano Marco Jakimowski Polacco, lo que haría tambalear las coordenadas históricas y editoriales. «As no original copy of the 1623 publication has survived, it remains unclear whether the mutiny occurred» ${ }^{36}$. Ante esta nueva perspectiva y siguiendo esta tesis, el acontecimiento habría sucedido antes y relato habría aparecido uno o dos años después, para reimprimirse cinco años más tarde en Roma, pero no lo sabemos con seguridad y por eso las referencias temporales resultan tan confusas. No sabemos, por tanto, si el evento se produjo antes de 1623 o en 1627.

b) Reliquia A. Holobutsky en Miroslav Mamchack, «Marco Jakimowski» y reliquia del evento. Indica que «El texto italiano [...] fue creado más tarde y tiene una diferencia muy notable: aquí la llegada de la galera está fechada el 7 de diciembre de 1622», mientras que la inscripción en latín en la que se basa, en honor a la patrona de Palermo y que aún se conserva en la iglesia de Santa Rosalía, indica la llegada en 1627. La traducción al español sería la siguiente:

Los rutenos, desde hace tiempo esclavos de los turcos, aconsejados y conducidos por Marco Jakimowski, alejada su galera militar de las otras tres, prepararon un escape muy hábil y perspicaz; obtenida la libertad y habiendo desembarcado en Palermo el 7 de diciembre de 1627 y en honor a la victoria sobre los turcos y la Santa Virgen, erigieron una galera como monumento en una roca; después de haber encontrado trabajo en la ciudad, han donado parte del botín para edificar una capilla dedicada a la santa patrona de Sicilia.

De nuevo, otro testimonio más que desmiente la versión de Marnavitio y que, además, resulta confuso al encontrar distintas fechas.

c) Relato alternativo B. Distinto año de llegada a Roma en Sadık Paşa (2015). Aquí nos encontramos con lo siguiente: «they left the captured galley in the possession of the Viceroy of Naples in exchange for two smaller vessels, which they took to Naples and on to Rome, where they arrived on February $16^{\text {th }}, 1621$ ». Otro año más a considerar. Tomasz Święcki, autor de Historia de la antigua Polonia (1621) también apuesta por esta fecha ${ }^{37}$. 
d) Relato alternativo C. Stanislaw Zieliński ¿1620 o 1621? En el diccionario sobre polacos pioneros sobre el mar de Zieliński (1933) existen dos entradas dedicadas a los dos compañeros con nombres italianizados y mencionados en las relaciones: Giovanni Stolcina y Stefano Sattanouski. En primer lugar, ofrece una consideración curiosa respecto al primero: «junto a Marek Jakimowski, obtuvo una galera turca en Mitilene en 1620»38. En segundo lugar, respecto a Sattanouski, curiosamente, se indica que «junto a Marek Jakimoswki y Stolcina obtuvo una galera turca en 1621 en Mitilene» ${ }^{39}$. También está la del propio Marek, pero en una obra del mismo autor dos años más tarde (Zieliński, 1935), donde la captura es en 1620 y la liberación es en $1621^{40}$.

Ante este panorama de indicaciones cronológicas diversas, solo podemos establecer las siguientes hipótesis con sus correspondientes consecuencias:

\begin{tabular}{|c|l|}
\hline Relato/reliquia & \multicolumn{1}{|c|}{ Cronología } \\
\hline Relato A & $\begin{array}{l}\text { Relación prínceps en } 1623 \text { que supuestamente } \\
\text { está perdida. }\end{array}$ \\
\hline Reliquia A & $\begin{array}{l}\text { Inscripción latina en Palermo que indica la lle- } \\
\text { gada de Marco en } 1627 \text { o el } 7 \text { de diciembre de } \\
1622 .\end{array}$ \\
\hline Relato B & Llegada a Roma el 16 de febrero de 1621. \\
\hline Relato C & $\begin{array}{l}\text { Stolcina captura la galera en 1620; Sattanouski, } \\
\text { en 1621. } \\
\text { Jakimowski es capturado en Jotín (Ucrania) en } \\
\text { 1620 y se libera en 1621. }\end{array}$ \\
\hline
\end{tabular}

Los resultados contrastan mucho con lo indicado por la relación de Marnavitio y sus traducciones (florentina, polaca y alemana), por no comentar la barcelonesa, que destaca sobre estas mismas con un cronotopo totalmente diferente y contradictorio. Además, una de las consecuencias de aceptar los relatos C, D o E sería también que Marco habría sido recibido por el papa Pablo III y no por Urbano VIII, que comienza su actividad en 1623.

Mostremos ahora las posibles hipótesis:

1) Historia de Marnavitio (1627-1628).

2) Historia de Santa Cruz (1628).

3) Suceso acaecido en 1620, 1621, 1622 o 1623. 
4) Jakimowski es uno de los cautivos que pediría un rescate en la batalla de Cécora.

5) Pura invención bien basándose en un caso particular, bien totalmente imaginada como literatura antiturca.

\section{CONCLUSIONES. ¿RECONSTRUCCIÓN HISTÓRICA?}

Como hemos apuntado al principio, los fenómenos históricos no pueden anclarse en un pasado remoto sin posibilidad de progresión hacia el presente. Es necesario recoger los relatos (las ediciones encontradas y asumir la posibilidad de la existencia de otras) y las reliquias (la inscripción latina) con las que contemos para tratar de delimitar dicho acontecimiento y convertirlo en un hecho más o menos estable desde el juicio crítico del historiador y/o filólogo, puesto que el rango científico del campo categorial de la Historia no puede permitirse la supresión del sujeto que opera sobre sus materiales y los reinterpreta inevitablemente desde distintas coordenadas. Es necesario, en resumen, tomar partido siempre de la forma más racional y lógica. Por otro lado, esto no quiere decir que vayamos a proceder a una reconstrucción histórica total, sino más bien parcial por la falta de documentación y de aclaración por parte de los historiadores y estudiosos en el asunto. Bastará exponer una hipótesis más para hacer progresar el estudio y la investigación de este fenómeno tan sonado y que pudo haber determinado varios imaginarios colectivos del momento gracias a su clarísima eficacia propagandística.

Ante las distintas variantes, semejanzas y diferencias que hemos ido encontrando, y ante la gran falta de información sobre el fenómeno y su protagonista, podemos señalar, en base a las hipótesis establecidas en el apartado anterior, que probablemente el suceso ocurriera muy próximo a la batalla de Cécora (1620) y podríamos confiar más en la inscripción de la iglesia de Palermo, pero la diferencia de fechas entre el texto en latín y su traducción en italiano no nos permite fijar esa asunción. Lo más razonable sería pensar que la historia de Jakimowski fuese una edulcoración ideológica de un hecho bastante menos épico que más adelante se retomaría, quizá porque Marnavitio o Grignani se enterasen (o promoviesen) de una prínceps en italiano o latín que no hemos podido localizar y decidieran reajustar los parámetros y adaptarla al contexto de 1628. Las características textuales divergentes y los testimonios dispares harían pensar en el uso de una leyenda de la que se apropia cada ámbito cultural (polaco-lituano, español, florentino, romano y alemán) englobado, a su vez, en la Iglesia Católica.

Decidiéndonos por una vía o no, lo que queda claro después de nuestra revisión y análisis documental es:

1) que las relaciones de sucesos desdibujan las líneas entre la Historia y la ficción;

2) que una relación de sucesos suele editarse varias veces y cada nueva versión puede poner en riesgo al hecho histórico tratado;

3) que la reconstrucción parcial de un hecho histórico puede establecer las bases para estudios futuros y su mejoramiento o bien estancamiento si no aparecen más fuentes reveladoras: 
4) que el relato fue, sin duda, aprovechado para construir la identidad nacional polaca durante el siglo XIX.

5) y que la épica cristiana en las relaciones de sucesos fue un fenómeno totalmente vigente a finales del siglo XVI y principios del XVII al servicio del poder eclesiástico y totalmente apóloga de territorios no pertenecientes al Imperio Otomano. De ahí la necesidad del presentismo histórico para comprender la dialéctica actual entre la Europa occidental y el Islam.

\section{BiBLIOGRAFÍA}

Alvar, Carlos, Traducciones y traductores. Materiales para una historia de la traducción en Castilla durante la Edad Media, Alcalá de Henares, Centro de Estudios Cervantinos, 2009.

BIDISO. Biblioteca Digital Siglo de Oro (1996-2021). Recuperado el 24/02/2021 de http://www.bidiso.es/.

Bilinski, Bronislaw, «220 schiavi cristiani liberati e portati a Roma nel 1628», en Manlio Barberito et al., Strenna dei Romanisti 1980, Roma, Editrice Roma Amor, 1980, pp. 77-91.

BNF. Bibliothèque National de France. Recuperado el 24/02/2021 de https://data. bnf.fr/fr/12230590/lodovico_grignani/.

Boffa, Elisa, «Una tipografia celata ad Arezzo: Francesco Onofri e Angelo Raimondi», Annali Aretini, XIV, 2006, pp. 41-54.

Chaulet, Rudy, «¿Dónde están los esclavos? Relaciones de sucesos y esclavitud: entre tópicos literarios y lagunas informativas», en La invención de las noticias. Las relaciones de sucesos entre la literatura y la información (siglos XVIXVIII), ed. Giovanni Ciappelli y Valentina Nider, Trento, Collana Labirinti (Università degli Studi di Trento), 2017, pp. 353-373.

Ciappelli, Giovanni, «L'informazione e la propaganda. La guerra di corsa delle galee toscane contro turchi e barbareschi nel Seicento, attraverso relazioni e relaciones a stampa», en La invención de las noticias. Las relaciones de sucesos entre la literatura y la información (siglos XVI-XVIII), ed. Giovanni Ciappelli y Valentina Nider, Trento, Collana Labirinti (Università degli Studi di Trento), 2017, pp. 133-161.

Delgado Casado, Juan, Diccionario de impresores españoles (Siglos XV-XVII), Madrid, Arco Libros, 1996.

Digital Library of Polish and Poland-Related News Pamphlets. Recuperado el 24/02/2021 de https://cbdu.ijp.pan.pl/.

«Incursiones crimeanas principios siglo XVII», Arre caballo!, 2019, https://arrecaballo.es/edad-moderna/guerras-ruso-crimeanas-1507-1689/incursionescrimeanas-principios-siglo-xvii/. 
Internet Culturale. Cataloghi e collezioni delle biblioteche italiane. Recuperado el 25/02/2021 de http://www.internetculturale.it/.

Lafarga, Francisco, y Pegenaute, Luis, Historia de la traducción en España, Salamanca, Editorial Ambos Mundos, 2004.

Legge e riforma del magistrato et ufizio de'pupilli et adulti della città di Firenze, Florencia, Francesco Onofri, 1680.

López Poza, Sagrario, «Relaciones de sucesos traducidas al español», en Géneros editoriales y relaciones de sucesos en la Edad Moderna, ed. María Eugenia Díaz Tena, SEMYR (Universidad de Salamanca), 2013, pp. 249-273.

Magosci, Paul, «The Rusyn Question», Political Thought, 2-3.6, 1995, pp. 221-231, http://www.litopys.org.ua/rizne/magocie.htm.

Mamchack, Miroslav, «Marco Jakimowski», Svitlytsia Crimea, 2021, s. p. http:// svitlytsia.crimea.ua/?section=article\&artID $=22978$.

Marnavitio, Marco, Relazione della conquista della galera capitana d'Alessandria nel porto di Metellino per opera del capitano Marco Jakimowski..., Roma, Ludovico Grignani, 1628.

Marnavitio, Marco, Relazione della conquista della galera capitana d'Alessandria nel porto di Metellino per opera del capitano Marco Jakimowski..., Florencia, Francesco Onofri, 1628.

Mazzuchelli, Giammaria, Gli scrittori d'Italia cioe' notizie storiche, e critiche intorno alle vite, e agli scritti dei letterati italiani del conte Giammaria Mazzuchelli bresciano Volume I. Parte I, Brescia, Giambattista Bossini, 1758.

Opisanie krótkie zdobycia galery aleksandryjskiej w porcie Metellino za sprawą kapitana Marka Jakimowskiego, Cracovia, s. i., 1628.

Paşa, Sadık, «Mutiny on the Kadırğa», Polonia Ottomanica, 2015, s. p., http://poloniaottomanica.blogspot.com/2014/04/mutiny-on-kadrga.html.

Pena Sueiro, Nieves, «Algunas consideraciones sobre el itinerario y la difusión de la literatura informativa en el Siglo de Oro, con el ejemplo de una relación de milagros», Artifara, 11, 2011, pp. 45-54.

Polczynski, Michael, «A Polish Slave's Escape from an Ottoman Galley», Docblog. Ottoman History Podcast, 2014, s. p., http://www.docblog.ottomanhistorypodcast.com/.

Quadrio, Francesco Saverio, Della storia e della ragione d'ogni poesia. Volume quattro, Milano, Francisco Agnelli, 1749.

Redondo, Agustín, «El mundo turco a través de las relaciones de sucesos de finales del S. XVI y de las primeras décadas del S. XVII: la percepción de la alteridad y su puesta en obra narrativa», en Encuentro de civilizaciones (1500-1750). Informar, narrar, celebrar, ed. Antonina Paba, Alcalá de Henares, Universidad de Alcalá (Servicio de Publicaciones), 2003, pp. 235-255. 
Ryba, Renata, Literatura staropolska wobec zjawiska niewoli tatarsko-tureckiej. Studia i szkice, Katowice, Wydawnictwo, Uniwersytetu Śląskiego, 2014.

Sandrigo, Alessandra, «Tipografi ed editori italiani del secolo XVII», Biblioteca Statale Isontina di Gorizia, 2019, s. p., https://www.isontina.beniculturali.it/it/488/ tipografi-ed-editori-italiani-del-secolo-xvii.

Santa Cruz, Miguel de, Relacion del verdadero levantamiento, y presa de la Galera Capitana de Alexandria, en el Puerto de Metellin, por industria del Capitan Marco Lachimosch Esclauo de dicha Galera, con libertad de 220. Christianos Esclauos, à 18.de Iulio 1628. Impressa ya en Roma. Traduzida de lengua Italiana en Castellano, por Miguel de Santa Cruz, Barcelona, Esteban Liberòs, 1628.

Ślaski, Boleslaw, Opanowanie w roku 1627 przez Marka Jakimowskiego okrętu tureckiego, Poznań, Czcionkami Drukarni Sw. Wojciecha, 1927.

Staniul, Michal, «Marek Jakimowski, un esclavo de la República de Polonia, se rebeló y liberó a 200 cristianos», 2015, s. p., https://opinie.wp.pl/marek-jakimowski-niewolnik-z-rzeczpospolitej-wzniecil-bunt-i-uwolnil-200-chrzescijan$6126039553660545 a$.

Torrentino, Lorenzo, Annali della tipografia fiorentina, Florencia, Biblioteca Nazionale Centrale di Firenze, 1819.

TRECCANI. II portal del sapere (1925-2021). Recuperado el 24/02/2021 de https:// www.treccani.it/.

Varriale, Gennaro, «¿La frontera mediterránea laboratorio de América? El espionaje contra el Turco en el siglo XVI», Cuadernos de Historia Moderna, 45.1, 2020, pp. 81-109.

Verwunderlicher Seehafenkrieg, das ist wahhafte Neue Zeitung wasmassen ein geborener Pole, aber gefangener Sklave, eine türkische Hauptgaleere durch seine Tapferkeit bewältigt und mit grossem Reichtum in die Christenheit gebracht hat, s. I., s. i., 1628.

Zieliński, Stanislaw, Mały słownik pionierów polskich kolonialnych i morskich: podróżnicy, odkrywcy, zdobywcy, badacze, eksploratorzy, emigranci - pamiętnikarze, działacze i pisarze migracyjni, Varsovia, Inst. Wyd. Ligi Morskiej i KoIonialnej, 1933, https://dbc.wroc.pl/dlibra/publication/10293/edition/9266/ maly-slownik-pionierow-polskich-kolonjalnych-i-morskich-podroznicy-odkrywcy-zdobywcy-badacze-eksploratorzy-emigranci-pamietnikarze-dzialacze-i-pisarze-migracyjni-zielinski-stanislaw-1880-1936?language=pl.

Zieliński, Stanislaw, Wybitne czyny Polaków na obczyźnie, Wilno, Światowy Związek Polaków z Zagranicy, 1935, http://bibliotekacyfrowa.eu/dlibra/show-content/ publication/48200/edition/42302/?format_id=2. 